\title{
Some Results on N-Tupled Coincidence and Fixed Points of Graphs on Metric Spaces and an Application to Integral Equations
}

\author{
Ahmed H. Soliman $\mathbb{D}^{1,2}$ and Tamer Nabil $\mathbb{D}^{1,3}$ \\ ${ }^{1}$ King Khalid University, College of Science, Department of Mathematics, P.O. Box 9004, 61413, Abha, Saudi Arabia \\ ${ }^{2}$ Department of Mathematics, Faculty of Science, Al-Azhar University, Assiut Branch, Assiut 71524, Egypt \\ ${ }^{3}$ Suez Canal University, Faculty of Computers and Informatics, Department of Basic Science, Ismailia, Egypt
}

Correspondence should be addressed to Ahmed H. Soliman; mahmod@kku.edu.sa

Received 17 September 2018; Revised 14 October 2018; Accepted 24 October 2018; Published 5 November 2018

Academic Editor: Richard I. Avery

Copyright (C) 2018 Ahmed H. Soliman and Tamer Nabil. This is an open access article distributed under the Creative Commons Attribution License, which permits unrestricted use, distribution, and reproduction in any medium, provided the original work is properly cited.

\begin{abstract}
In this work, we establish some $\mathrm{N}$-tupled common coincidence and $\mathrm{N}$-tupled common fixed points for the mappings satisfying a $(\varphi-\psi)$-type contractive condition in a complete metric space endowed with a directed graph (for short digraph). Also, we apply our theoretical results to study the existence and uniqueness of solutions for systems of integral equations.
\end{abstract}

\section{Introduction}

The theory of fixed point has become very important and useful to solve many mathematical problems in different subjects of sciences such as dynamical programming [1], optimization theory [2], signal processing [3], and iterative process [4], and other times it is used to prove the existence of solution of differential and integral equations $[5,6]$.

Coupled fixed point was started by Guo and Lakshmikantham [7] in 1987 which is additionally trailed by Bhasker and Lakshmikantham [8] wherein authors gave the notion of mixed monotone property for a generalized contraction mapping $F: X^{2} \longrightarrow X$ (where $X$ is an ordered metric space) and utilized the same to introduce some results on existence of coupled fixed points which are seen as coupled definition of specific results of Nieto and Lopez [9]. In 2009, Lakshmikantham and Ciric [10] extended these results for nonlinear contraction mappings by giving the notions of mixed g-monotone property and coupled coincidence point. Recently, the idea of coupled fixed point is reached out to higher dimensions by numerous authors. In fact, in 2010, Samet and Vetro [11] defined the notion of fixed point of n-tupled fixed point, $n \in \mathbb{N}$ and $n \geq 2$, as natural extension of coupled fixed point and proved some theorems for $\mathrm{n}$-tupled fixed point in complete metric spaces. In 2013, Imdad et al. [12] extended the notion of n-tupled fixed point for a pair of mappings $\mathrm{F}$ and $\mathrm{g}$ by defining $\mathrm{n}$ tupled coincidence point and also introduced the general concept of mixed g-monotone property on $X^{n}$ for merely even $n$. Also, they employed the same to prove an even-tupled coincidence theorem for nonlinear contractions satisfying mixed g-monotone property. In fact, this result is true for only even $n$ but not for odd ones. Recently, Kir et al. [13] considered the existence of a coupled fixed point of $(\varphi, \psi)$-contractive mappings endowed with a digraph and applied the obtained results to the system of two integral equations. There already exists an extensive literature on this topic. Keeping in view the relevance of this paper, we refer to [14-21].

In this paper, we will obtain more general results of Kir et al. [13], which are common $\mathrm{N}$-tupled coincidence points and common N-tupled fixed points of operators satisfying $(\varphi, \psi)$-contractive condition in metric spaces endowed with a digraph.

Our paper is organized as follows. In Section 2, some basic definitions are introduced. Section 3 contains some results on $\mathrm{N}$-tupled coincidence points. In Section 4, the existence of a common $\mathrm{N}$-tupled fixed point of operators 
satisfying $(\varphi, \psi)$-contractive conditions in metric spaces endowed with a digraph is considered. Section 5 gives an application to integral equations to support availability of the obtained results.

\section{Preliminaries}

Through all of this paper let $(X, d)$ be a complete metric space. We recall the following definition which is given in [22].

Definition 1. An element $\left(x^{1}, x^{2}, \ldots, x^{N}\right) \in X^{N}$ is said to be an N-tupled fixed point of the function $F: X^{N} \longrightarrow X$ if

$$
\begin{gathered}
F\left(x^{1}, x^{2}, \ldots, x^{N}\right)=x^{1}, \\
F\left(x^{2}, x^{3}, \ldots, x^{1}\right)=x^{2}, \\
\vdots \\
F\left(x^{N}, x^{2}, \ldots, x^{N-1}\right)=x^{N} .
\end{gathered}
$$

Therefore, an element $\left(x^{1}, x^{2}, \ldots, x^{N}\right) \in X^{N}$ is said to be a common $N$-tupled coincidence point of the functions $F$ : $X^{N} \longrightarrow X$ and $g: X \longrightarrow X$ if

$$
\begin{gathered}
F\left(x^{1}, x^{2}, \ldots, x^{N}\right)=g x^{1} \\
F\left(x^{2}, x^{3}, \ldots, x^{1}\right)=g x^{2} \\
\vdots \\
F\left(x^{N}, x^{2}, \ldots, x^{N-1}\right)=g x^{N} .
\end{gathered}
$$

Also, an element $\left(x^{1}, x^{2}, \ldots, x^{N}\right) \in X^{N}$ is said to be a common N-tupled fixed point of the functions $F: X^{N} \longrightarrow X$ and $g: X \longrightarrow X$ if $\left(x^{1}, x^{2}, \ldots, x^{N}\right)$ is N-tupled fixed point for both $F$ and $g$.

The following definition of commutative mappings in $X^{N}$ is the generalization of the definition of Lakshmikntham and Ciric [10].

Definition 2. The two mappings $F: X^{N} \longrightarrow X$ and $g: X \longrightarrow$ $X$ are said to be commutative if

$$
g F\left(x^{1}, x^{2}, \ldots, x^{N}\right)=F\left(g x^{1}, g x^{2}, \ldots, g x^{N}\right)
$$

for all $\left(x^{1}, x^{2}, \ldots, x^{N}\right) \in X^{N}$.

Inspired by the definition of compatible mappings in $X^{2}$ of Choudhury and Kundu [23], we introduce the following definition.
Definition 3. The mappings $F: X^{N} \longrightarrow X$ and $g: X \longrightarrow X$ are called compatible if

$$
\begin{gathered}
\lim _{n \rightarrow \infty} d\left(g\left(F\left(x_{n}^{1}, x_{n}^{2}, \ldots, x_{n}^{N}\right)\right),\right. \\
\left.F\left(g x_{n}^{1}, g x_{n}^{2}, \ldots, g x_{n}^{N}\right)\right)=0, \\
\lim _{n \rightarrow \infty} d\left(g\left(F\left(x_{n}^{2}, x_{n}^{3}, \ldots, x_{n}^{N}, x_{n}^{1}\right)\right),\right. \\
\left.F\left(g x_{n}^{2}, g x_{n}^{3}, \ldots, g x_{n}^{N}, g x_{n}^{1}\right)\right)=0, \\
\vdots \\
\lim _{n \rightarrow \infty} d\left(g\left(F\left(x_{n}^{N}, x_{n}^{1}, \ldots, x_{n}^{N-1}\right)\right),\right. \\
\left.F\left(g x_{n}^{N}, g x_{n}^{1}, \ldots, g x_{n}^{N-1}\right)\right)=0,
\end{gathered}
$$

where $\left\{x_{n}^{1}\right\},\left\{x_{n}^{2}\right\}, \ldots,\left\{x_{n}^{N}\right\}$ are sequences in $X$ such that

$$
\begin{gathered}
\lim _{n \rightarrow \infty} F\left(x_{n}^{1}, x_{n}^{2}, \ldots, x_{n}^{N}\right)=\lim _{n \rightarrow \infty} g\left(x_{n}^{1}\right)=x^{1}, \\
\vdots \\
\lim _{n \longrightarrow \infty} F\left(x_{n}^{N}, x_{n}^{1}, \ldots, x_{n}^{N-1}\right)=\lim _{n \longrightarrow \infty} g\left(x_{n}^{N}\right)=x^{N},
\end{gathered}
$$

where $\left(x^{1}, x^{2}, \ldots, x^{N}\right) \in X^{N}$.

Definition 4 (see [24]). Let $\Phi$ be the set of all functions $\varphi$ : $[0, \infty) \longrightarrow[0, \infty)$ such that, for any $\varphi \in \Phi$, all following conditions are satisfied:

(1) $\varphi$ is lower semi-continuous and (strictly) increasing;

(2) $\varphi(t)<t$ for all $t>0$;

(3) $\varphi(t+s) \leq \varphi(t)+\varphi(s)$ for all $t, s \in[0, \infty)$;

one can note that $\lim _{n \rightarrow \infty} \varphi\left(t_{n}\right)=0$ if and only if $\lim _{n \rightarrow \infty} t_{n}=0$ for $t_{n} \in[0, \infty)$;

(4) for $\varphi \in \Phi, \Psi_{\varphi}$ denote all functions $\psi:[0, \infty) \longrightarrow$ $[0, \infty)$ which satisfy the following:

(4A) $\limsup _{n \longrightarrow \infty} \psi\left(t_{n}\right)<\varphi(r)$ if $\lim _{n \longrightarrow \infty} t_{n}=r>0$;

(4B) $\lim _{n \rightarrow \infty} \psi\left(t_{n}\right)=0$ if $\lim _{n \rightarrow \infty} t_{n}=0$ for a sequence $\left\{t_{n}\right\} \in[0, \infty)$.

Now, let $(X, d)$ be a metric space. Define $\triangle=\{(x, x): x \in X\}$ to be a diagonal of $X^{2}$ and $G=(V(G), E(G))$ is digraph which does not have parallel edges, where $V(G)$ is the set of vertices of $G$ and $E(G)$ is the set of edges of $G$.

Through all of this paper, let $V(G)$ coincide with $X$ and $\triangle \in E(G)$. Chifu and Petrusel [25] considered the concept of $G$-continuous mappings which is extended in $X^{N}$ by the following definition. 
Definition 5. Let $F: X^{N} \longrightarrow X$ and $g: X \longrightarrow X$ be two mappings. Then (I) $F$ is said to be $G-N$-continuous if for all $\left(x^{1 *}, x^{2 *}, \ldots, x^{N *}\right) \in X^{N}$ and any positive sequence $\left\{n_{i}\right\}_{i} \in \mathbb{N}$

$$
\begin{gathered}
F\left(x_{n_{i}}^{1}, x_{n_{i}}^{2}, \ldots, x_{n_{i}}^{N}\right) \longrightarrow x^{1 *}, \\
F\left(x_{n_{i}}^{2}, x_{n_{i}}^{3}, \ldots, x_{n_{i}}^{1}\right) \longrightarrow x^{2 *}, \\
\vdots \\
F\left(x_{n_{i}}^{N}, x_{n_{i}}^{1}, \ldots, x_{n_{i}}^{N-1}\right) \longrightarrow x^{N *},
\end{gathered}
$$

as $i \longrightarrow \infty$ and

$$
\begin{aligned}
& \left(F\left(x_{n_{i}}^{1}, x_{n_{i}}^{2}, \ldots, x_{n_{\mathrm{i}}}^{N}\right), F\left(x_{n_{i+1}}^{1}, x_{n_{i+1}}^{2}, \ldots, x_{n_{i+1}}^{N}\right)\right) \\
& \quad \in E(G) ; \\
& \left(F\left(x_{n_{i}}^{2}, x_{n_{i}}^{3}, \ldots, x_{n_{i}}^{1}\right), F\left(x_{n_{i+1}}^{2}, x_{n_{i+1}}^{3}, \ldots, x_{n_{i+1}}^{1}\right)\right) \\
& \quad \in E(G), \\
& \vdots \\
& \left(F\left(x_{n_{i}}^{N}, x_{n_{i}}^{1}, \ldots, x_{n_{i}}^{N-1}\right), F\left(x_{n_{i+1}}^{N}, x_{n_{i+1}}^{1}, \ldots, x_{n_{i+1}}^{N-1}\right)\right) \\
& \quad \in E(G) .
\end{aligned}
$$

We have

$$
\begin{gathered}
F\left(F\left(x_{n_{i}}^{1}, x_{n_{i}}^{2}, \ldots, x_{n_{i}}^{N}\right), F\left(x_{n_{i}}^{2}, x_{n_{i}}^{3}, \ldots, x_{n_{i}}^{1}\right), \ldots,\right. \\
\left.F\left(x_{n_{i}}^{N}, x_{n_{i}}^{1}, \ldots, x_{n_{i}}^{N-1}\right)\right)=F\left(x^{1 *}, x^{2 *}, \ldots, x^{N *}\right), \\
F\left(F\left(x_{n_{i}}^{2}, x_{n_{i}}^{3}, \ldots, x_{n_{i}}^{1}\right), F\left(x_{n_{i}}^{3}, x_{n_{i}}^{4}, \ldots, x_{n_{i}}^{(2)}\right), \ldots,\right. \\
\left.F\left(x_{n_{i}}^{1}, x_{n_{i}}^{2}, \ldots, x_{n_{i}}^{N}\right)\right)=F\left(x^{2 *}, x^{3 *}, \ldots, x^{1 *}\right), \\
\vdots \\
F\left(F\left(x_{n_{i}}^{N}, x_{n_{i}}^{1}, \ldots, x_{n_{i}}^{N-1}\right), F\left(x_{n_{i}}^{1}, x_{n_{i}}^{2}, \ldots, x_{n_{i}}^{N}\right), \ldots,\right. \\
\left.F\left(x_{n_{i}}^{N-1}, x_{n_{i}}^{N}, \ldots, x_{n_{i}}^{(1)}\right)\right)=F\left(x^{N *}, x^{1 *}, \ldots, x^{N-1 *}\right) .
\end{gathered}
$$

(II) $(X, d, G)$ has property $A$ if any sequence $\left\{x_{n}\right\} \subseteq X$ with $x_{n} \longrightarrow x$ as $n \longrightarrow \infty$ and $\left(x_{n}, x_{n+1}\right) \in E(G)$, then $\left(x_{n}, x\right) \in$ $E(G)$.

Consider the set $\operatorname{NTCP}(F, g)$ of all N-tupled coincidence points of the mappings $F: X^{N} \longrightarrow X$ and $g: X \longrightarrow X$, i.e.,

$$
\begin{aligned}
& \operatorname{NTCP}(F, g)=\left\{\left(x^{1}, x^{2}, \ldots, x^{N}\right)\right. \\
& \quad \in X^{N}: F\left(x^{1}, x^{2}, \ldots, x^{N}\right)=g x^{1}, F\left(x^{2}, x^{3}, \ldots, x^{1}\right) \\
& \left.\quad=g x^{2}, \ldots, F\left(x^{N}, x^{1}, \ldots, x^{N-1}\right)=g x^{N}\right\} .
\end{aligned}
$$

Therefore, $\left(X^{N}\right)_{g}^{F}$ is defined as

$$
\begin{aligned}
& \left(X^{N}\right)_{g}^{F}=\left\{\left(x^{1}, x^{2}, \ldots, x^{N}\right)\right. \\
& \quad \in X^{N}:\left(g x^{1}, F\left(x^{1}, x^{2}, \ldots, x^{N}\right)\right) \\
& \left.\quad \in E(G), \ldots,\left(g x^{N}, F\left(x^{N}, x^{1}, \ldots, x^{N-1}\right)\right) \in E(G)\right\} .
\end{aligned}
$$

In 2015, Suantai et al. [26] considered the definition of $G$ - edge preserving which is extended by the following definition.

Definition 6. The mappings $F: X^{N} \longrightarrow X$ and $g: X \longrightarrow X$ are said to be $N-G$-edge preserving if

$$
\left[\left(g x^{1}, g u^{1}\right), \ldots,\left(g x^{N}, g u^{N}\right)\right] \in E(G)
$$

implies that

$$
\begin{aligned}
& {\left[\left(F\left(x^{1}, \ldots, x^{N}\right), F\left(u^{1}, \ldots, u^{N}\right)\right), \ldots,\right.} \\
& \left.\quad\left(F\left(x^{N}, \ldots, X^{N-1}\right), F\left(u^{N}, \ldots, u^{N-1}\right)\right)\right] \in E(G) .
\end{aligned}
$$

Definition 7 (see [26]). $E(G)$ satisfies the transitivity property in $X$ if and only if $\forall x, y, t \in X$, where $(x, y),(y, t) \in E(G) \longrightarrow$ $(x, t) \in E(G)$.

\section{An N-Tupled Coincidence Point Theorem}

Firstly, we give the following definition which will be used in our main results.

Definition 8 . Let $(X, d)$ be a complete metric space endowed with digraph $G$. The mappings $F: X^{N} \longrightarrow X$ and $g: X \longrightarrow$ $X$ are called $(\phi, \psi)$-contractive if

(i) $F$ and $f$ are $N-G$ - edge preserving,

(ii) there exist $\varphi \in \Phi$ and $\psi \in \Psi_{\varphi}$ such that, for all $\left(x^{1}, \ldots, x^{N}\right)$ and $\left(u^{1}, \ldots, u^{N}\right) \in X^{N}$,

$$
\left(g x^{1}, g u^{1}\right), \ldots,\left(g x^{N}, g u^{N}\right) \in E(G),
$$

then

$$
\begin{aligned}
\varphi & \left(2 ^ { - 1 } \left\{d\left(F\left(x^{1}, \ldots, x^{N}\right), F\left(u^{1}, \ldots, u^{N}\right)\right)\right.\right. \\
& +d\left(F\left(x^{2}, \ldots, x^{1}\right), F\left(u^{2}, \ldots, u^{1}\right)\right)+\cdots \\
& \left.\left.+d\left(F\left(x^{N}, \ldots, x^{N-1}\right), F\left(u^{N}, \ldots, u^{N-1}\right)\right)\right\}\right) \\
& \leq \psi\left(d\left(g x^{1}, g u^{1}\right)+d\left(g x^{2}, g u^{2}\right)+\cdots\right. \\
& \left.+d\left(g x^{N}, g u^{N}\right)\right) .
\end{aligned}
$$

We study the existence of $\mathrm{N}$-tupled coincidence point of the mappings $F: X^{N} \longrightarrow X$ and $g: X \longrightarrow X$ under the following hypotheses:

(H1) $g$ is continuous and $g(X)$ is closed;

(H2) $F\left(X^{N}\right) \subseteq g(X)$, and $(F, g)$ is compatible;

(H3) $F$ is $N-G$-continuous;

(H4) the tripled $(X, d, G)$ has a property $A$.

Now, we are ready to introduce our $N$-tupled coincidence point theorem. 
Theorem 9. Let $F: X^{N} \longrightarrow X$ and $g: X \longrightarrow X$ be $(\varphi, \psi)$-contractive mappings, where $(X, d)$ is a complete metric space and $G$ is the digraph endowed by $X$. If the hypotheses $(H 1),(H 2)$, and $(H 3)$ hold, then $\operatorname{NTCP}(F, g) \neq \emptyset$ if and only if $\left(X^{N}\right)_{g}^{F} \neq \emptyset$.

Proof. Consider $\left(x_{0}^{1}, x_{0}^{2}, \ldots, x_{0}^{N}\right),\left(y_{0}^{1}, y_{0}^{2}, \ldots, y_{0}^{N}\right) \in X^{N}$. Since $F\left(X^{N}\right) \subseteq g(X)$, then there exist

$x_{1}^{1}, x_{1}^{2}, \ldots, x_{1}^{N}, y_{1}^{1}, y_{1}^{2}, \ldots, y_{1}^{N} \in X$ such that

$$
\begin{gathered}
F\left(x_{0}^{1}, x_{0}^{2}, \ldots, x_{0}^{N}\right)=g x_{1}^{1} \\
F\left(x_{0}^{2}, x_{0}^{3}, \ldots, x_{0}^{1}\right)=g x_{1}^{2} \\
\vdots \\
F\left(x_{0}^{N}, x_{0}^{1}, \ldots, x_{0}^{N-1}\right)=g x_{1}^{N}
\end{gathered}
$$

Also, we have that

$$
\begin{gathered}
F\left(y_{0}^{1}, y_{0}^{2}, \ldots, y_{0}^{N}\right)=g y_{1}^{1} \\
F\left(y_{0}^{2}, y_{0}^{3}, \ldots, y_{0}^{1}\right)=g y_{1}^{2} \\
\vdots \\
F\left(y_{0}^{N}, y_{0}^{1}, \ldots, y_{0}^{N-1}\right)=g y_{1}^{N} .
\end{gathered}
$$

By doing this process, then we get the sequences $\left\{x_{n}^{1}\right\}$, $\left\{x_{n}^{2}\right\}, \ldots,\left\{x_{n}^{N}\right\},\left\{y_{n}^{1}\right\},\left\{y_{n}^{2}\right\}, \ldots,\left\{y_{n}^{N}\right\}$ such that

$$
\begin{gathered}
F\left(x_{n}^{1}, x_{n}^{2}, \ldots, x_{n}^{N}\right)=g x_{n+1}^{1} ; \\
F\left(x_{n}^{2}, x_{n}^{3}, \ldots, x_{n}^{1}\right)=g x_{n+1}^{2} ; \\
\vdots \\
F\left(x_{n}^{N}, x_{n}^{1}, \ldots, x_{n}^{N-1}\right)=g x_{n+1}^{N} .
\end{gathered}
$$

Also, we have that

$$
\begin{gathered}
F\left(y_{n}^{1}, y_{n}^{2}, \ldots, y_{n}^{N}\right)=g y_{n+1}^{1} ; \\
F\left(y_{n}^{2}, y_{n}^{3}, \ldots, y_{n}^{1}\right)=g y_{n+1}^{2} ; \\
\vdots \\
F\left(y_{n}^{N}, y_{n}^{1}, \ldots, y_{n}^{N-1}\right)=g y_{n+1}^{N} .
\end{gathered}
$$

Now, suppose that $\operatorname{NTCP}(F, g) \neq \emptyset$. Let $\left(u^{1}, u^{2}, \ldots, u^{N}\right) \epsilon$ $\operatorname{NTCP}(F, g)$. Thus, we have that

$$
\begin{gathered}
F\left(u^{1}, u^{2}, \ldots, u^{N}\right)=g u^{1} \\
F\left(u^{2}, u^{3}, \ldots, u^{1}\right)=g u^{2} \\
\vdots \\
F\left(u^{N}, u^{1}, \ldots, u^{N-1}\right)=g u^{N} .
\end{gathered}
$$

Hence, we get that

$$
\begin{aligned}
& \left(g u^{1}, F\left(u^{1}, u^{2}, \ldots, u^{N}\right)\right)=\left(g u^{1}, g u^{1}\right) \in E(G) ; \\
& \left(g u^{2}, F\left(u^{2}, u^{3}, \ldots, u^{1}\right)\right)=\left(g u^{2}, g u^{2}\right) \in E(G) ;
\end{aligned}
$$

$$
\left(g u^{N}, F\left(u^{N}, u^{1}, \ldots, u^{N-1}\right)\right)=\left(g u^{N}, g u^{N}\right) \in E(G) .
$$

Hence, $\left(u^{1}, u^{2}, \ldots, u^{N}\right) \in\left(X^{N}\right)_{g}^{F}$. Then, we obtain $\left(X^{N}\right)_{g}^{F} \neq \emptyset$. Let $\left(x_{0}^{1}, \ldots, x_{0}^{N}\right) \in\left(X^{N}\right)_{g}^{F}$, then we have that

$$
\begin{aligned}
& \left(g x_{0}^{1}, F\left(x_{0}^{1}, x_{0}^{2}, \ldots, x_{0}^{N}\right)\right) \in E(G) ; \\
& \left(g x_{0}^{2}, F\left(x_{0}^{2}, x_{0}^{3}, \ldots, x_{0}^{1}\right)\right) \in E(G) ;
\end{aligned}
$$

$$
\left(g x_{0}^{N}, F\left(x_{0}^{N}, x_{0}^{1}, \ldots, x_{0}^{N-1}\right)\right) \in E(G) .
$$

By using (15), we get that

$$
\begin{gathered}
\left(g x_{0}^{1}, F\left(x_{0}^{1}, x_{0}^{2}, \ldots, x_{0}^{N}\right)\right)=\left(g x_{0}^{1}, g x_{1}^{1}\right) \in E(G) ; \\
\left(g x_{0}^{2}, F\left(x_{0}^{2}, x_{0}^{3}, \ldots, x_{0}^{1}\right)\right)=\left(g x_{0}^{2}, g x_{1}^{2}\right) \in E(G) ; \\
\vdots \\
\left(g x_{0}^{N}, F\left(x_{0}^{N}, x_{0}^{1}, \ldots, x_{0}^{N-1}\right)\right)=\left(g x_{0}^{N}, g x_{1}^{N}\right) \in E(G) .
\end{gathered}
$$

Since $F$ and $g$ are $N-G$-edge preserving, we have that

$$
\begin{aligned}
& \left(F\left(x_{0}^{1}, x_{0}^{2}, \ldots, x_{0}^{N}\right), F\left(x_{1}^{1}, x_{1}^{2}, \ldots, x_{1}^{N}\right)\right) \\
& \quad=\left(g x_{1}^{1}, g x_{2}^{1}\right) \in E(G) ; \\
& \left(F\left(x_{0}^{2}, x_{0}^{3}, \ldots, x_{0}^{1}\right), F\left(x_{1}^{2}, x_{1}^{3}, \ldots, x_{1}^{1}\right)\right)=\left(g x_{1}^{2}, g x_{2}^{2}\right) \\
& \quad \in E(G) ; \\
& \quad \vdots \\
& \left(F\left(x_{0}^{N}, x_{0}^{1}, \ldots, x_{0}^{N-1}\right), F\left(x_{1}^{N}, x_{1}^{1}, \ldots, x_{1}^{N-1}\right)\right) \\
& \quad=\left(g x_{1}^{N}, g x_{2}^{N}\right) \in E(G) .
\end{aligned}
$$

By doing this process, we have

$$
\begin{aligned}
& {\left[\left(g x_{n}^{1}, g x_{n+1}^{1}\right),\left(g x_{n}^{2}, g x_{n+1}^{2}\right), \ldots,\left(g x_{n}^{N}, g x_{n+1}^{N}\right)\right]} \\
& \quad \in E(G) .
\end{aligned}
$$

Let $r_{n}=\left(\left(d\left(g x_{n+1}^{1}, g x_{n+2}^{1}\right)+d\left(g x_{n+1}^{2}, g x_{n+2}^{2}\right)+\cdots+\right.\right.$ $\left.\left.d\left(g x_{n+1}^{N}, g x_{n+2}^{N}\right)\right) / 2\right)$. We get that 


$$
\begin{aligned}
& \varphi\left(\frac{d\left(g x_{n+1}^{1}, g x_{n+2}^{1}\right)+d\left(g x_{n+1}^{2}, g x_{n+2}^{2}\right)+\cdots+d\left(g x_{n+1}^{N}, g x_{n+2}^{N}\right)}{2}\right) \\
& =\varphi\left(\frac{d\left(F\left(x_{n}^{1}, \ldots, x_{n}^{N}\right), F\left(x_{n+1}^{1}, \ldots, x_{n+1}^{N}\right)\right)+d\left(F\left(x_{n}^{2}, \ldots, x_{n}^{1}\right), F\left(x_{n+1}^{2}, \ldots, x_{n+1}^{1}\right)\right)+\cdots+d\left(F\left(x_{n}^{N}, \ldots, x_{n}^{N-1}\right), F\left(x_{n+1}^{N}, \ldots, x_{n+1}^{N-1}\right)\right)}{2}\right) \\
& \leq \psi\left(\frac{d\left(g x_{n}^{1}, g x_{n+1}^{1}\right)+d\left(g x_{n}^{2}, g x_{n+1}^{2}\right)+\cdots+d\left(g x_{n}^{N}, g x_{n+1}^{N}\right)}{2}\right),
\end{aligned}
$$

for all $n \in \mathbb{N}$, then we have that

$$
\varphi\left(r_{n+1}\right) \leq \psi\left(r_{n}\right)<\varphi\left(r_{n}\right)
$$

By using (26) and because $\varphi$ is monotonic function, we obtain $\left\{r_{n}\right\}$ is a positive decreasing sequence. Then, $r_{n} \longrightarrow r$ as $n \longrightarrow$ $\infty$ for some $r \in[0, \infty)$. Consider $r>0$. In (26), taking the limit as $n \longrightarrow \infty$ and from the properties of $\varphi, \psi$, we have that

$$
\varphi(r) \leq \lim \sup _{n \longrightarrow \infty} \varphi\left(r_{n+1}\right) \leq \lim \sup _{n \longrightarrow \infty} \psi\left(r_{n}\right)<\varphi(r)
$$

We obtain contradiction. Thus $r=0$ and then we get

$$
\lim _{n \rightarrow \infty} r_{n}=\lim _{n \rightarrow \infty}\left(\frac{d\left(g x_{n}^{1}, g x_{n+1}^{1}\right)+d\left(g x_{n}^{2}, g x_{n+1}^{2}\right)+\cdots+d\left(g x_{n}^{N}, g x_{n+1}^{N}\right)}{2}\right)=0
$$

Now, we will show that $\left\{g x_{n}^{1}\right\},\left\{g x_{n}^{2}\right\}, \ldots,\left\{g x_{n}^{N}\right\}$ are Cauchy sequences. Let $\left\{g x_{n}^{1}\right\},\left\{g x_{n}^{2}\right\}, \ldots,\left\{g x_{n}^{N}\right\}$ be not Cauchy sequences. Then there exist $\epsilon>0$, positive integers $n(k)>m(k) \geq k$ and we can find $\left\{g x_{n(k)}^{1}\right\},\left\{g x_{m(k)}^{1}\right\},\left\{g x_{n(k)}^{2}\right\},\left\{g x_{m(k)}^{2}\right\}, \ldots,\left\{g x_{n(k)}^{N}\right\},\left\{g x_{m(k)}^{N}\right\}$ such that

$$
\eta_{k}=\frac{\left(d\left(g x_{n(k)}^{1}, g x_{m(k)}^{1}\right)+d\left(g x_{n(k)}^{2}, g x_{m(k)}^{2}\right)+\cdots+d\left(g x_{n(k)}^{N}, g x_{m(k)}^{N}\right)\right)}{2} \geq \epsilon .
$$

Therefore, we can find $n(k)$ is the smallest integer for which (29) holds, and we have that

$$
\frac{\left(d\left(g x_{n(k)-1}^{1}, g x_{m(k)}^{1}\right)+d\left(g x_{n(k)-1}^{2}, g x_{m(k)}^{2}\right)+\cdots+d\left(g x_{n(k)-1}^{N}, g x_{m(k)}^{N}\right)\right)}{2} \geq \epsilon .
$$

Using (29) and (30), we get

$$
\epsilon \leq \eta_{k}<\epsilon+\frac{\left(d\left(g x_{n(k)}^{1}, g x_{n(k)-1}^{1}\right)+d\left(g x_{n(k)}^{2}, g x_{n(k)-1}^{2}\right)+\cdots+d\left(g x_{n(k)}^{N}, g x_{n(k)-1}^{N}\right)\right)}{2} .
$$

From (27) and (31), we get that

$$
\eta_{k}=\frac{d\left(g x_{n(k)}^{1}, g x_{m(k)}^{1}\right)+d\left(g x_{n(k)}^{2}, g x_{m(k)}^{2}\right)+\cdots+d\left(g x_{n(k)}^{N}, g x_{m(k)}^{N}\right)}{2} \longrightarrow 0, \text { as } k \longrightarrow \infty
$$


Therefore, we obtain that

$$
\begin{aligned}
\eta_{k} & =\frac{\left(d\left(g x_{n(k)}^{1}, g x_{m(k)}^{1}\right)+d\left(g x_{n(k)}^{2}, g x_{m(k)}^{2}\right)+\cdots+d\left(g x_{n(k)}^{N}, g x_{m(k)}^{N}\right)\right)}{2} \\
& \leq r_{n(k)}+r_{m(k)}+\frac{\left(d\left(g x_{n(k)+1}^{1}, g x_{m(k)+1}^{1}\right)+d\left(g x_{n(k)+1}^{2}, g x_{m(k)+1}^{2}\right)+\cdots+d\left(g x_{n(k)+1}^{N}, g x_{m(k)+1}^{N}\right)\right)}{2} .
\end{aligned}
$$

Using the properties of $\varphi$, we get that

$$
\begin{aligned}
\varphi\left(\eta_{k}\right) \leq & \varphi\left(r_{n(k)}\right)+\varphi\left(r_{m(k)}\right)+\varphi\left(\frac{\left(d\left(g x_{n(k)+1}^{1}, g x_{m(k)+1}^{1}\right)+d\left(g x_{n(k)+1}^{2}, g x_{m(k)+1}^{2}\right)+\cdots+d\left(g x_{n(k)+1}^{N}, g x_{m(k)+1}^{N}\right)\right)}{2}\right) \\
\leq & \varphi\left(r_{n(k)}\right)+\varphi\left(r_{m(k)}\right) \\
& +\varphi\left(\frac{\left(d\left(F\left(x_{n(k)}^{1}, \ldots, x_{n(k)}^{N}\right), F\left(x_{m(k)}^{1}, \ldots, x_{m(k)}^{N}\right)\right)+\cdots+d\left(F\left(x_{n(k)}^{N}, \ldots, x_{n(k)}^{N-1}\right), F\left(x_{m(k)}^{N}, \ldots, x_{m(k)}^{N-1}\right)\right)\right)}{2}\right) \\
\leq & \varphi\left(r_{n(k)}\right)+\varphi\left(r_{m(k)}\right)+\psi\left(\frac{\left.d\left(g x_{n(k)}^{1}, g x_{m(k)}^{1}\right)+\cdots+d\left(g x_{n(k)}^{N}, g x_{m(k)}^{N}\right)\right)}{2}\right) \leq \varphi\left(r_{n(k)}\right)+\varphi\left(r_{m(k)}\right)+\varphi\left(\eta_{k}\right) .
\end{aligned}
$$

Again from the properties of $\varphi$ which is lower semicontinuous, if we take the limit as $k \longrightarrow \infty$, we have that

$$
\begin{aligned}
\varphi(\epsilon) \leq & \lim _{k \rightarrow \infty} \sup _{k \rightarrow \infty}\left(\eta_{n(k)}\right) \\
\leq & \lim _{k \rightarrow \infty} \varphi\left(r_{n(k)}\right)+\lim _{k \rightarrow \infty} \varphi\left(r_{m(k)}\right) \\
& +\lim _{k \rightarrow \infty} \sup _{k \rightarrow \infty} \psi\left(\eta_{n(k)}\right)<\varphi(\epsilon) .
\end{aligned}
$$

That is a contradiction. Hence, $\left\{g x_{n}^{1}\right\},\left\{g x_{n}^{2}\right\}, \ldots,\left\{g x_{n}^{N}\right\}$ are Cauchy sequences. Then, there exist $u^{1}, u^{2}, \ldots, u^{N} \in g(X)$ such that

$$
\begin{gathered}
\lim _{n \longrightarrow \infty} F\left(x_{n}^{1}, x_{n}^{2}, \ldots, x_{n}^{N}\right)=\lim _{n \longrightarrow \infty} g x_{n}^{1}=u^{1} ; \\
\lim _{n \longrightarrow \infty} F\left(x_{n}^{2}, x_{n}^{3}, \ldots, x_{n}^{1}\right)=\lim _{n \longrightarrow \infty} g x_{n}^{2}=u^{2} ; \\
\vdots \\
\lim _{n \rightarrow \infty} F\left(x_{n}^{N}, x_{n}^{1}, \ldots, x_{n}^{N-1}\right)=\lim _{n \longrightarrow \infty} g x_{n}^{N}=u^{N} .
\end{gathered}
$$

Since $(F, g)$ is compatible, we get

$$
\begin{aligned}
& \lim _{n \rightarrow \infty} d\left(g\left(F\left(x_{n}^{1}, x_{n}^{2}, \ldots, x_{n}^{N}\right)\right),\right. \\
& \left.\quad F\left(g x_{n}^{1}, x_{n}^{2}, \ldots, g x_{n}^{N}\right)\right)=0 \\
& \lim _{n \rightarrow \infty} d\left(g\left(F\left(x_{n}^{2}, x_{n}^{3}, \ldots, x_{n}^{1}\right)\right), F\left(g x_{n}^{2}, x_{n}^{3}, \ldots, g x_{n}^{1}\right)\right)
\end{aligned}
$$

$$
=0
$$$$
\begin{gathered}
\lim _{n \longrightarrow \infty} d\left(g\left(F\left(x_{n}^{N}, x_{n}^{1}, \ldots, x_{n}^{N-1}\right)\right),\right. \\
\left.F\left(g x_{n}^{N}, x_{n}^{1}, \ldots, g x_{n}^{N-1}\right)\right)=0 .
\end{gathered}
$$

Since $F$ is $N-G$-continuous, we get, for all $n \geq 0$,

$$
\begin{aligned}
& \left.d\left(F\left(g x_{n}^{1}, x_{n}^{2}, \ldots, g x_{n}^{N}\right)\right), g u^{1}\right) \\
& \left.\quad \leq d\left(F\left(g x_{n}^{1}, x_{n}^{2}, \ldots, g x_{n}^{N}\right)\right), g F\left(x_{n}^{1}, x_{n}^{2}, \ldots, x_{n}^{N}\right)\right) \\
& \quad+d\left(g F\left(x_{n}^{1}, x_{n}^{2}, \ldots, x_{n}^{N}, g u^{1}\right) .\right. \\
& \left.d\left(F\left(g x_{n}^{2}, x_{n}^{3}, \ldots, g x_{n}^{1}\right)\right), g u^{2}\right) \\
& \left.\quad \leq d\left(F\left(g x_{n}^{2}, x_{n}^{3}, \ldots, g x_{n}^{1}\right)\right), g F\left(x_{n}^{2}, x_{n}^{3}, \ldots, x_{n}^{1}\right)\right) \\
& \quad+d\left(g F\left(x_{n}^{2}, x_{n}^{3}, \ldots, x_{n}^{1}\right), g u^{2}\right) .
\end{aligned}
$$

$$
\begin{aligned}
& \left.d\left(F\left(g x_{n}^{N}, x_{n}^{1}, \ldots, g x_{n}^{N-1}\right)\right), g u^{N}\right) \\
& \quad \leq d\left(F\left(g x_{n}^{N}, x_{n}^{1}, \ldots, g x_{n}^{N-1}\right)\right), \\
& \left.g F\left(x_{n}^{N}, x_{n}^{1}, \ldots, x_{n}^{N-1}\right)\right) \\
& \quad+d\left(g F\left(x_{n}^{N}, x_{n}^{1}, \ldots, x_{n}^{N-1}\right), g u^{N}\right) .
\end{aligned}
$$


Taking the limit as $n \longrightarrow \infty$ and using (H1) and (H2), we obtain that

$$
d\left(F\left(u^{1}, u^{2}, \ldots, u^{N}\right), g u^{1}\right)=0, d\left(F\left(u^{2}, u^{3}, \ldots, u^{1}\right), g u^{2}\right)=0, \ldots, d\left(F\left(u^{N}, u^{1}, \ldots, u^{N-1}\right), g u^{N}\right)=0
$$

Thus, we have that

$$
F\left(u^{1}, u^{2}, \ldots, u^{N}\right)=g u^{1}, F\left(u^{2}, u^{3}, \ldots, u^{1}\right)=g u^{2}, \ldots, F\left(u^{N}, u^{1}, \ldots, u^{N-1}\right)=g u^{N}
$$

Hence, $\operatorname{NTCP}(F, g) \neq \emptyset$.

Theorem 10. Let $F: X^{N} \longrightarrow X$ and $g: X \longrightarrow X$ be $(\varphi, \psi)$-contractive mappings, where $(X, d)$ is a complete metric space and $G$ is the digraph endowed by $X$. If the hypotheses $(H 1),(H 2)$, and $(H 4)$ hold, then $\operatorname{NTCP}(F, g) \neq \emptyset$ if and only if $\left(X^{N}\right)_{g}^{F} \neq \emptyset$.

Proof. Let $\left(u^{1}, \ldots, u^{N}\right),\left(x^{1}, \ldots, x^{N}\right) \in X^{N}$ such that $u^{1}=$ $g x^{1}, \ldots, u^{N}=g x^{N}$. Following the same argument as in the proof of Theorem 9 , we get sequences $\left\{x_{n}^{1}\right\}, \ldots,\left\{x_{n}^{N}\right\}$ such that

$$
\left[\left(g x_{n}^{1}, x^{1}\right), \ldots,\left(g x_{n}^{N}, x^{N}\right)\right] \in E(G) .
$$

Thus, we have that

$$
\begin{aligned}
& d\left(g x^{1}, F\left(x^{1}, \ldots, x^{N}\right)\right) \leq d\left(g x^{1}, g x_{n+1}^{1}\right) \\
& \quad+d\left(g x_{n+1}^{1}, F\left(x^{1}, \ldots, x^{N}\right)\right)=d\left(g x^{1}, g x_{n+1}^{1}\right) \\
& \quad+d\left(F\left(x_{n}^{1}, \ldots, x_{n}^{N}\right), F\left(x^{1}, \ldots, x^{N}\right)\right) ; \\
& d\left(g x^{2}, F\left(x^{2}, \ldots, x^{1}\right)\right) \leq d\left(g x^{2}, g x_{n+1}^{2}\right) \\
& \quad+d\left(g x_{n+1}^{2}, F\left(x^{2}, \ldots, x^{1}\right)\right)=d\left(g x^{2}, g x_{n+1}^{2}\right)
\end{aligned}
$$

$$
\begin{gathered}
+d\left(F\left(x_{n}^{2}, \ldots, x_{n}^{1}\right), F\left(x^{2}, \ldots, x^{1}\right)\right) \\
\vdots \\
d\left(g x^{N}, F\left(x^{N}, \ldots, x^{N-1}\right)\right) \leq d\left(g x^{N}, g x_{n+1}^{N}\right) \\
+d\left(g x_{n+1}^{N}, F\left(x^{N}, \ldots, x^{N-1}\right)\right)=d\left(g x^{N}, g x_{n+1}^{N}\right) \\
+d\left(F\left(x_{n}^{N}, \ldots, x_{n}^{N-1}\right), F\left(x^{N}, \ldots, x^{N-1}\right)\right) .
\end{gathered}
$$

Thus, we obtain that

$$
\begin{gathered}
d\left(g x^{1}, F\left(x^{1}, \ldots, x^{N}\right)\right)-d\left(g x^{1}, g x_{n+1}^{1}\right) \\
\leq d\left(F\left(x_{n}^{1}, \ldots, x_{n}^{N}\right), F\left(x^{1}, \ldots, x^{N}\right)\right) ; \\
d\left(g x^{2}, F\left(x^{2}, \ldots, x^{1}\right)\right)-d\left(g x^{2}, g x_{n+1}^{2}\right) \\
\leq d\left(F\left(x_{n}^{2}, \ldots, x_{n}^{1}\right), F\left(x^{2}, \ldots, x^{1}\right)\right) ; \\
\vdots \\
d\left(g x^{N}, F\left(x^{N}, \ldots, x^{N-1}\right)\right)-d\left(g x^{N}, g x_{n+1}^{N}\right) \\
\leq d\left(F\left(x_{n}^{N}, \ldots, x_{n}^{N-1}\right), F\left(x^{N}, \ldots, x^{N-1}\right)\right),
\end{gathered}
$$

which implies that

$$
\begin{gathered}
\frac{d\left(g x^{1}, F\left(x^{1}, \ldots, x^{N}\right)\right)-d\left(g x^{1}, g x_{n+1}^{1}\right)+\cdots+d\left(g x^{N}, F\left(x^{N}, \ldots, x^{N-1}\right)\right)-d\left(g x^{N}, g x_{n+1}^{N}\right)}{2} \\
\leq \frac{d\left(F\left(x_{n}^{1}, \ldots, x_{n}^{N}\right), F\left(x^{1}, \ldots, x^{N}\right)\right)+\cdots+d\left(F\left(x_{n}^{N}, \ldots, x_{n}^{N-1}\right), F\left(x^{N}, \ldots, x^{N-1}\right)\right)}{2} .
\end{gathered}
$$


Again using the monotonicity property of $\varphi$, we get

$$
\begin{aligned}
& \varphi\left(\frac{d\left(g x^{1}, F\left(x^{1}, \ldots, x^{N}\right)\right)-d\left(g x^{1}, g x_{n+1}^{1}\right)+\cdots+d\left(g x^{N}, F\left(x^{N}, \ldots, x^{N-1}\right)\right)-d\left(g x^{N}, g x_{n+1}^{N}\right)}{2}\right) \\
& \quad \leq \varphi\left(\frac{d\left(F\left(x_{n}^{1}, \ldots, x_{n}^{N}\right), F\left(x^{1}, \ldots, x^{N}\right)\right)+\cdots+d\left(F\left(x_{n}^{N}, \ldots, x_{n}^{N-1}\right), F\left(x^{N}, \ldots, x^{N-1}\right)\right)}{2}\right) \\
& \quad \leq \psi\left(\frac{d\left(g x_{n}^{1}, g x^{1}\right)+\cdots+d\left(g x_{n}^{N}, g x^{N}\right)}{2}\right) .
\end{aligned}
$$

By taking the limit as $n \longrightarrow \infty$, we obtain that

$$
g x^{1}=F\left(x^{1}, \ldots, x^{N}\right), \ldots, g x^{N}=F\left(x^{N}, \ldots, x^{1}\right) .
$$

Hence, $\operatorname{NTCP}(F, g) \neq \emptyset$.

\section{The Existence and Uniqueness of N-Tupled Fixed Points}

Let the set of all common N-tupled fixed points of the mappings $F: X^{N} \longrightarrow X$ and $g: X \longrightarrow X$ denoted by $\operatorname{NTF}(F, g)$ and defined as

$$
\begin{array}{r}
\operatorname{NTF}(F, g)=\left\{\left(x^{1}, \ldots, x^{N}\right) \in X^{N}: F\left(x^{1}, \ldots, X^{N}\right)\right. \\
\left.\quad=g x^{1}=x^{1}, \ldots, F\left(x^{N}, \ldots, x^{N-1}\right)=g x^{N}=x^{N}\right\} .
\end{array}
$$

Now, we consider the following fixed point property:

$(F P)$ Whenever $F: X^{N} \longrightarrow X$ and $g: X \longrightarrow X$ are two mappings, then $F, g$ have a unique common N-tupled fixed point.

Theorem 11. Let $F: X^{N} \longrightarrow X$ and $g: X \longrightarrow X$ be commutative $(\varphi, \psi)$-contractive mappings, where $(X, d)$ is a complete metric space and $G$ is the digraph endowed by $X$. If the hypotheses (H1), (H2), and (H3) hold and for any two elements $\left(x^{1}, \ldots, x^{N}\right),\left(y^{1}, \ldots, y^{N}\right) \in X^{N}$ there exists $\left(z^{1}, \ldots, z^{N}\right) \in X^{N}$ such that $\left[\left(g x^{1}, g z^{1}\right),\left(g y^{1}, g z^{1}\right)\right.$, $\left.\ldots,\left(g x^{N}, g z^{N}\right),\left(g y^{N}, g z^{N}\right)\right] \in E(G)$. Then $\left(X^{N}\right)_{g}^{F} \neq \emptyset$ if and only if $F, g$ have the fixed point property (FP).

Proof. By doing the same argument as in the proof of Theorem 9 , there exists $\left(x^{1}, x^{2}, \ldots, x^{N}\right) \in X^{N}$ such that

$$
\begin{gathered}
F\left(x^{1}, x^{2}, \ldots, x^{N}\right)=g x^{1} \\
F\left(x^{2}, x^{3}, \ldots, x^{1}\right)=g x^{2} \\
\vdots \\
F\left(x^{N}, x^{1}, \ldots, x^{N-1}\right)=g x^{N} .
\end{gathered}
$$

Assume there exists another element $\left(y^{1}, y^{2}, \ldots, y^{N}\right) \in X^{N}$ such that

$$
\begin{gathered}
F\left(y^{1}, y^{2}, \ldots, y^{N}\right)=g y^{1} \\
F\left(y^{2}, y^{3}, \ldots, y^{1}\right)=g y^{2} \\
\vdots \\
F\left(y^{N}, y^{1}, \ldots, y^{N-1}\right)=g y^{N} .
\end{gathered}
$$

We will show that $g x^{1}=g y^{1}, g x^{2}=g y^{2}, \ldots, g x^{N}=g y^{N}$. We can find $\left(z^{1}, z^{2}, \ldots, z^{N}\right) \in X^{N}$ such that

$$
\begin{aligned}
& {\left[\left(g x^{1}, g z^{1}\right),\left(g y^{1}, g z^{1}\right), \ldots,\left(g x^{N}, g z^{N}\right),\left(g y^{N}, g z^{N}\right)\right]} \\
& \quad \in E(G) .
\end{aligned}
$$

By doing the same process as in (17) and (18), we get the sequences $\left\{x_{n}^{1}\right\}, \ldots,\left\{x_{n}^{N}\right\},\left\{y_{n}^{1}\right\}, \ldots,\left\{y_{n}^{N}\right\},\left\{z_{n}^{1}\right\}, \ldots,\left\{z_{n}^{N}\right\}$ such that

$$
\begin{aligned}
& x^{1}=x_{0}^{1}, x^{2}=x_{0}^{2}, \ldots, x^{N}=x_{0}^{N} ; \\
& y^{1}=y_{0}^{1}, y^{2}=y_{0}^{2}, \ldots, y^{N}=y_{0}^{N}
\end{aligned}
$$

and

$$
z^{1}=z_{0}^{1}, z^{2}=z_{0}^{2}, \ldots, z^{N}=z_{0}^{N}
$$

Therefore, we obtain that

$$
\begin{gathered}
F\left(x_{n}^{1}, x_{n}^{2}, \ldots, x_{n}^{N}\right)=g x_{n+1}^{1} ; \\
F\left(x_{n}^{2}, x_{n}^{3}, \ldots, x_{n}^{1}\right)=g x_{n+1}^{2} \\
\vdots \\
F\left(x_{n}^{N}, x_{n}^{1}, \ldots, x_{n}^{N-1}\right)=g x_{n+1}^{N} \\
F\left(y_{n}^{1}, y_{n}^{2}, \ldots, y_{n}^{N}\right)=g y_{n+1}^{1} ;
\end{gathered}
$$




$$
\begin{gathered}
F\left(y_{n}^{2}, y_{n}^{3}, \ldots, y_{n}^{1}\right)=g y_{n+1}^{2} ; \\
\vdots \\
F\left(y_{n}^{N}, y_{n}^{1}, \ldots, y_{n}^{N-1}\right)=g y_{n+1}^{N} ; \\
F\left(z_{n}^{1}, z_{n}^{2}, \ldots, z_{n}^{N}\right)=g z_{n+1}^{1} ; \\
F\left(z_{n}^{2}, z_{n}^{3}, \ldots, z_{n}^{1}\right)=g z_{n+1}^{2} ; \\
\vdots \\
F\left(z_{n}^{N}, z_{n}^{1}, \ldots, z_{n}^{N-1}\right)=g z_{n+1}^{N} ;
\end{gathered}
$$

and we obtain that

$$
\begin{aligned}
& F\left(x^{1}, x^{2}, \ldots, x^{N}\right)=g x_{n}^{1} \\
& F\left(x^{2}, x^{3}, \ldots, x^{1}\right)=g x_{n}^{2} ; \\
& F\left(x^{N}, x^{1}, \ldots, x^{N-1}\right)=g x_{n}^{N} \\
& F\left(y^{1}, y^{2}, \ldots, y^{N}\right)=g y_{n}^{1} \\
& F\left(y^{2}, y^{3}, \ldots, y^{1}\right)=g y_{n}^{2} ; \\
& F\left(y^{N}, y^{1}, \ldots, y^{N-1}\right)=g y_{n}^{N} \\
& \varphi\left(\frac{d\left(g z_{n+1}^{1}, g x^{1}\right)+\cdots+d\left(g z_{n+1}^{N}, g x^{N}\right)}{2}\right) \\
& \leq \varphi\left(\frac{d\left(F\left(z_{n}^{1}, \ldots, z_{n}^{N}\right), F\left(x^{1}, \ldots, x^{N}\right)\right)+\cdots+d\left(F\left(z_{n}^{N}, \ldots, z_{n}^{N-1}\right), F\left(x^{N}, \ldots, x^{N-1}\right)\right)}{2}\right) \\
& \leq \psi\left(\frac{d\left(g z_{n}^{1}, g x^{1}\right)+\cdots+d\left(g z_{n}^{N}, g x^{N}\right)}{2}\right) . \\
& \left(F\left(x^{N}, x^{1}, \ldots, x^{N-1}\right), F\left(z_{0}^{N}, z_{0}^{1}, \ldots, z_{0}^{N-1}\right)\right) \\
& =\left(g x^{N}, g z_{1}^{N}\right) \in E(G) . \\
& \left(F\left(x^{1}, x^{2}, \ldots, x^{N}\right), F\left(z_{0}^{1}, z_{0}^{2}, \ldots, z_{0}^{N}\right)\right)=\left(g x^{1}, g z_{1}^{1}\right) \\
& \in E(G) \text {; } \\
& \left(F\left(x^{2}, x^{3}, \ldots, x^{1}\right), F\left(z_{0}^{3}, z_{0}^{2}, \ldots, z_{0}^{1}\right)\right)=\left(g x^{2}, g z_{1}^{2}\right) \\
& \in E(G) \text {; }
\end{aligned}
$$

therefore, we get that
We obtain that

$$
\varphi\left(\xi_{n+1}\right) \leq \psi\left(\xi_{n}\right),
$$

where

$$
\xi_{n}=\frac{d\left(g z_{n}^{1}, g x^{1}\right)+\cdots+d\left(g z_{n}^{N}, g x^{N}\right)}{2} .
$$

The sequence $\left\{\xi_{n}\right\}$ is monotonic decreasing sequence of positive real numbers; then $\exists \xi \geq 0$ such that

$$
\xi_{n} \longrightarrow \infty \text { as } n \longrightarrow \infty \text {. }
$$

Let $\xi>0$. Take the limit as $n \longrightarrow \infty$ for (59),

$$
\varphi(\xi) \leq \lim _{n \rightarrow \infty} \sup _{n \rightarrow \infty} \varphi\left(\xi_{n+1}\right)<\varphi(\xi) .
$$

This is a contradiction; hence $\xi=0$. Therefore,

$$
\lim _{n \longrightarrow \infty} \frac{d\left(g z_{n}^{1}, g x^{1}\right)+\cdots d\left(g z_{n}^{N}, g x^{N}\right)}{2}=0,
$$


which gives that

$$
\begin{aligned}
\lim _{n \rightarrow \infty} d\left(g z_{n}^{1}, g x^{1}\right) & =\lim _{n \longrightarrow \infty} d\left(g z_{n}^{2}, g x^{2}\right)=\cdots \\
& =\lim _{n \longrightarrow \infty} d\left(g z_{n}^{N}, g x^{N}\right)=0 ;
\end{aligned}
$$

and according to $\left[\left(g z^{1}, g y^{1}\right), \ldots,\left(g z^{N}, g y^{N}\right)\right] \in E(G)$, then we get that

$$
\begin{aligned}
\lim _{n \longrightarrow \infty} d\left(g z_{n}^{1}, g y^{1}\right) & =\lim _{n \longrightarrow \infty} d\left(g z_{n}^{2}, g y^{2}\right)=\cdots \\
& =\lim _{n \longrightarrow \infty} d\left(g z_{n}^{N}, g y^{N}\right)=0 .
\end{aligned}
$$

Since

$$
d\left(g y^{1}, g x^{1}\right) \leq d\left(g y^{1}, g z_{n}^{1}\right)+d\left(g z_{n}^{1}, g x^{1}\right)
$$

for all $n \in \mathbb{N}$, then by taking the limit as $n \longrightarrow \infty$, we have that

$$
d\left(g y^{1}, g x^{1}\right)=d\left(g y^{2}, x^{2}\right)=\cdots=d\left(g y^{N}, x^{N}\right)=0 .
$$

Thus, $g x^{1}=g y^{1}, g x^{1}=g y^{2}, \ldots, g x^{N}=g y^{N}$. Hence the uniqueness of N-tupled common coincidence point for $F$ and $g$ is obtained. Let $\left(w^{1}, w^{2}, \ldots, w^{N}\right) \in X^{N}$ be the unique $\mathrm{N}$ tupled common coincidence point for $F$ and $g$, then it is clear that

$$
w^{1}=F\left(w^{1}, w^{2}, \ldots, w^{N}\right), w^{2}=F\left(w^{2}, w^{3}, \ldots, w^{1}\right), \ldots, w^{N}=F\left(w^{N}, w^{1}, \ldots, w^{N-1}\right)
$$

Since $(F, g)$ are commutative, we have

$$
g w^{1}=g F\left(w^{1}, w^{2}, \ldots, w^{N}\right)=F\left(g w^{1}, g w^{2}, \ldots, g w^{N}\right), g w^{2}=g F\left(w^{2}, w^{3}, \ldots, w^{1}\right)=F\left(g w^{2}, g w^{3}, \ldots, g, w^{1}\right), \ldots, g w^{N}=g F\left(w^{N}, w^{1}, \ldots, w^{N-1}\right)=F\left(g w^{N}, g w^{1}, \ldots, g w^{N-1}\right)
$$

Hence, $\left(g w^{1}, g w^{2}, \ldots, g w^{N}\right)$ is another N-tupled common coincidence point for $F$ and $g$. Then, we obtain that

$$
w^{1}=g w^{1}=F\left(w^{1}, w^{2}, \ldots, w^{N}\right), w^{2}=g w^{2}=F\left(w^{2}, w^{3}, \ldots, w^{1}\right), \ldots, w^{N}=g w^{N}=F\left(w^{N}, w^{1}, \ldots, w^{N-1}\right)
$$

To prove the uniqueness of $\mathrm{N}$-tupled fixed point, let $\left(\widehat{w^{1}}, \widehat{w^{2}}, \ldots, \widehat{w^{N}}\right) \in X^{N}$ be another fixed point. Then there exists $\left(u^{1}, u^{2}, \ldots, u^{N}\right) \in X^{N}$ such that

$$
u_{0}^{1}=u^{1}, u_{0}^{2}=u^{2}, \ldots, u_{0}^{N}=u^{N},
$$

and we obtain that

$$
\begin{aligned}
& F\left(u_{n}^{1}, u_{n}^{2}, \ldots, u_{n}^{N}\right)=g u_{n+1}^{1} \\
& F\left(u_{n}^{2}, u_{n}^{3}, \ldots, u_{n}^{1}\right)=g u_{n+1}^{2}
\end{aligned}
$$

$$
F\left(u_{n}^{N}, u_{n}^{1}, \ldots, u_{n}^{N-1}\right)=g u_{n+1}^{N}
$$

for all $n \in \mathbb{N}$. Then we have that

$$
\left[\left(g w^{1}, g u_{n}^{1}\right),\left(g w^{2}, g u_{n}^{2}\right), \ldots,\left(g w^{N}, u_{n}^{N}\right)\right] \in E(G) .
$$

Then we have that

$$
\begin{aligned}
& \varphi\left(\frac{d\left(w^{1}, g u_{n+1}^{1}\right)+\cdots+d\left(w^{2}, g u_{n+1}^{N}\right)}{2}\right) \\
& \quad=\varphi\left(\frac{d\left(F\left(w^{1}, \ldots, w^{N}\right), F\left(u_{n+1}^{1}, \ldots, u_{n+1}^{N}\right)\right)+\cdots+d\left(F\left(w^{N}, \ldots, w^{N-1}\right), F\left(u_{n+1}^{N}, \ldots, u_{n+1}^{N-1}\right)\right)}{2}\right) \\
& \quad \leq \psi\left(\frac{d\left(w_{n}^{1}, g u_{n}^{1}\right)+\cdots+d\left(w_{n}^{N}, g u_{n}^{N}\right)}{2}\right) .
\end{aligned}
$$


Therefore, by putting

$$
\rho_{n}=\frac{d\left(w_{n}^{1}, g u_{n}^{1}\right)+\cdots+d\left(w_{n}^{N}, g u_{n}^{N}\right)}{2},
$$

we obtain

$$
\varphi\left(\rho_{n+1}\right) \leq \psi\left(\rho_{n}\right)
$$

By doing the same steps above, we can show that there exist $\rho \geq 0$ such that $\rho_{n} \longrightarrow \rho$ as $n \longrightarrow \infty$. If $\rho>0$,

$$
\varphi(\rho) \leq \lim \sup _{n \longrightarrow \infty} \varphi\left(\rho_{n+1}\right) \leq \lim _{n \longrightarrow \infty} \psi\left(\rho_{n}\right)<\varphi(\rho),
$$

which is a contraction. Hence it must be $\rho=0$. Then we have that

$$
\begin{aligned}
\lim _{n \longrightarrow \infty} d\left(w^{1}, u_{n}^{1}\right) & =\lim _{n \longrightarrow \infty} d\left(w^{2}, u_{n}^{2}\right)=\cdots \\
& =\lim _{n \rightarrow \infty} d\left(w^{N}, u_{n}^{N}\right)=0 .
\end{aligned}
$$

Similarly, we can prove

$$
\begin{aligned}
\lim _{n \rightarrow \infty} d\left(\widehat{w^{1}}, u_{n}^{1}\right) & =\lim _{n \rightarrow \infty} d\left(\widehat{w^{2}}, u_{n}^{2}\right)=\cdots \\
& =\lim _{n \longrightarrow \infty} d\left(\widehat{w^{N}}, u_{n}^{N}\right)=0 .
\end{aligned}
$$

Hence, we get $\widehat{w^{1}}=w^{1}, \widehat{w^{2}}=w^{2}, \ldots, \widehat{w^{N}}=w^{N}$.

Theorem 12. Let $F: X^{N} \longrightarrow X$ and $g: X \longrightarrow X$ be commutative $(\varphi, \psi)$-contractive mappings, where $(X, d)$ is a complete metric space and $G$ is the digraph endowed by $X$. If the hypotheses (H1), (H2), and (H4) hold and for any two elements $\left(x^{1}, \ldots, x^{N}\right),\left(y^{1}, \ldots, y^{N}\right) \in$ $X^{N}$ there exist $\left(z^{1}, \ldots, z^{N}\right) \in X^{N}$ such that $\left[\left(g x^{1}, g z^{1}\right),\left(g y^{1}, g z^{1}\right), \ldots,\left(g x^{N}, g z^{N}\right),\left(g y^{N}, g z^{N}\right)\right] \in E(G)$, then $\left(X^{N}\right)_{g}^{F} \neq \emptyset$ if and only if $F, g$ have the fixed point property $(F P)$.

\section{An application}

Now, we apply the N-tupled fixed point theorem to study the existence and uniqueness of the solution of the system of nonlinear integral equations. This system can be written as

$$
\begin{gathered}
x_{1}(t)=A(t) \\
+\int_{0}^{a} B(t, s) K\left(s, x_{1}(s), x_{2}(s), \ldots, x_{N}(s)\right) d s ; \\
x_{2}(t)=A(t) \\
+\int_{0}^{a} B(t, s) K\left(s, x_{2}(s), x_{3}(s), \ldots, x_{1}(s)\right) d s ; \\
\quad: \\
x_{N}(t)=A(t) \\
+\int_{0}^{a} B(t, s) K\left(s, x_{N}(s), x_{1}(s), \ldots, x_{N-1}(s)\right) d s,
\end{gathered}
$$

where $a>0, t \in[0, a]$. Define the space $X=C\left([0, a], \mathfrak{R}^{n}\right)=$ $\left\{x:[0, a] \longrightarrow \mathfrak{R}^{n}: x\right.$ is continuous $\}$, where, for all $x \in X$, $\|x\|=\max _{t \in[0, a]}|x(t)|$. Therefore, $d(x, y)=\|x-y\|$ and $(X, d)$ is a complete metric space. Let $G$ be a digraph which $V(G)$ coincides with $X$ and $E(G)$ can be obtained by the the partial order relation

$$
(x, y) \in E(G) \Longleftrightarrow x \leq y \Longleftrightarrow x(t) \leq y(t)
$$

$$
\text { for all } t \in[0, a] \text {. }
$$

Thus $(X, d)$ is endowed with a digraph $G$. It is clear that the transitivity property is satisfied on $E(G)$ and $(x, d, G)$ has property $A$. To prove the existence of unique solution of (80), we consider

(C1) $K:[0, a] \times \underbrace{\mathfrak{R}^{N} \times \mathfrak{R}^{N} \ldots \times \mathfrak{R}^{N}}_{N \text {-times }} \longrightarrow \mathfrak{R}^{N}$ and $A:$ $[0, a] \longrightarrow \mathfrak{R}^{\mathrm{N}}$ are continuous,

(C2) there exists a $0 \leq \theta \leq 1$ such that

$$
\begin{aligned}
& \left|K\left(s, x_{1}, x_{2}, \ldots, x_{N}\right)-K\left(s, y_{1}, y_{2}, \ldots, y_{N}\right)\right| \\
& \quad \leq \theta\left(\left|x_{1}-y_{1}\right|+\left|x_{2}-y_{2}\right|+\cdots+\left|x_{N}-y_{N}\right|\right),
\end{aligned}
$$

(C3) $\forall s, t \in[0, a]$, there exists a continuous mapping $B$ : $[0, a] \times \mathfrak{R} \longrightarrow \mathfrak{R}$ such that

$$
\sup _{t \in[0, a]} \int_{0}^{a} B(t, s) d s<1,
$$

(C4) there exists $\left(x_{1}^{0}, x_{2}^{0}, \ldots, x_{N}^{0}\right) \in X^{N}$ such that

$$
\begin{aligned}
& x_{1}^{0}(t) \leq A(t) \\
& +\int_{0}^{a} B(t, s) K\left(s, x_{1}^{0}(s), x_{2}^{0}(s), \ldots, x_{N}^{0}(s)\right) d s ; \\
& x_{2}^{0}(t) \leq A(t) \\
& \quad+\int_{0}^{a} B(t, s) K\left(s, x_{2}^{0}(s), x_{3}^{0}(s), \ldots, x_{1}^{0}(s)\right) d s ;
\end{aligned}
$$

$$
\begin{aligned}
& x_{N}^{0}(t) \leq A(t) \\
& \quad+\int_{0}^{a} B(t, s) K\left(s, x_{N}^{0}(s), x_{1}^{0}(s), \ldots, x_{N-1}^{0}(s)\right) d s,
\end{aligned}
$$

for all $t \in[0, a]$.

Theorem 13. If conditions (C1), (C2), (C3), and (C4) are satisfied, then system (80) has a unique solution in $C\left([0, a], \mathfrak{R}^{n}\right)$.

Proof. Define $F: X^{N} \longrightarrow X$, where

$$
\begin{aligned}
& F\left(x_{1}, x_{2}, \ldots, x_{N}\right)(t) \\
& =A(t) \\
& \quad+\int_{0}^{a} B(t, s) K\left(s, x_{1}(s), x_{2}(s), \ldots, x_{N}(s)\right) d s ;
\end{aligned}
$$


Define $g: X \longrightarrow X$ as $g x(t)=2 x(t)$. System (80) can be written as

$$
x_{1}=F\left(x_{1}, x_{2}, \ldots, x_{N}\right), x_{2}=F\left(x_{2}, x_{3}, \ldots, x_{1}\right), \ldots, x_{N}=F\left(x_{N}, x_{1}, \ldots, x_{N-1}\right) .
$$

Let $x_{1}, x_{2}, \ldots, x_{N}, u_{1}, u_{2}, \ldots, u_{N} \in X$ such that

$$
g x_{1} \leq g u_{1}, g x_{2} \leq g u_{2}, \ldots, g x_{N} \leq g u_{N} .
$$

Then we get that $x_{1} \leq u_{1}, x_{2} \leq u_{2}, \ldots, x_{N} \leq u_{N}$, and

$$
\begin{aligned}
& F\left(x_{1}, x_{2}, \ldots, x_{N}\right)(t)=A(t) \\
& \quad+\int_{0}^{a} B(t, s) K\left(s, x_{1}(s), x_{2}(s), \ldots, x_{N}(s)\right) d s \\
& \quad \leq A(t) \\
& \quad+\int_{0}^{a} B(t, s) K\left(s, u_{1}(s), u_{2}(s), \ldots, u_{N}(s)\right) d s \\
& \quad=F\left(u_{1}, u_{2}, \ldots, u_{N}\right)(t) ; \\
& F\left(x_{2}, x_{3}, \ldots, x_{1}\right)(t)=A(t) \\
& \quad+\int_{0}^{a} B(t, s) K\left(s, x_{2}(s), x_{3}(s), \ldots, x_{1}(s)\right) d s
\end{aligned}
$$

$$
\begin{aligned}
& \leq A(t) \\
& +\int_{0}^{a} B(t, s) K\left(s, u_{2}(s), u_{3}(s), \ldots, u_{1}(s)\right) d s \\
& =F\left(u_{2}, u_{3}, \ldots, u_{1}\right)(t) ;
\end{aligned}
$$

$$
\begin{aligned}
F & \left(x_{N}, x_{1}, \ldots, x_{N-1}\right)(t)=A(t) \\
& +\int_{0}^{a} B(t, s) K\left(s, x_{N}(s), x_{1}(s), \ldots, x_{N-1}(s)\right) d s \\
& \leq A(t) \\
& +\int_{0}^{a} B(t, s) K\left(s, u_{N}(s), u_{1}(s), \ldots, u_{N-1}(s)\right) d s \\
& =F\left(u_{N}, u_{1}, \ldots, u_{N-1}\right)(t) ;
\end{aligned}
$$

for all $t \in[0, a]$

Hence, $(F, g)$ are N-G-edge preserving. By using conditions (C2) and (C3), and for all $t \in[0, a]$, we get that

$$
\begin{aligned}
& \frac{\left|F\left(x_{1}, \ldots, x_{N}\right)(t)-F\left(u_{1}, \ldots, u_{N}\right)(t)\right|+\cdots+\left|F\left(x_{N}, \ldots, x_{N-1}\right)(t)-F\left(u_{N}, \ldots, u_{N-1}\right)(t)\right|}{2} \\
& \quad=\frac{\left|\int_{0}^{a} B(t, s)\left[K\left(s, x_{1}(s), x_{2}(s), \ldots, x_{N}(s)\right)-K\left(s, u_{1}(s), u_{2}(s), \ldots, u_{N}(s)\right)\right] d s\right|}{2}+\cdots \\
& \quad+\frac{\left|\int_{0}^{a} B(t, s)\left[K\left(s, x_{N}(s), x_{1}(s), \ldots, x_{N-1}(s)\right)-K\left(s, u_{N}(s), u_{1}(s), \ldots, u_{N-1}(s)\right)\right] d s\right|}{2} \\
& \quad \leq \frac{\left|F\left(x_{1}, \ldots, x_{N}\right)(t)-F\left(u_{1}, \ldots, u_{N}\right)(t)\right|+\cdots+\left|F\left(x_{N}, \ldots, x_{N-1}\right)(t)-F\left(u_{N}, \ldots, u_{N-1}\right)(t)\right|}{2} \\
& \quad=\frac{\int_{0}^{a} B(t, s)\left|K\left(s, x_{1}(s), x_{2}(s), \ldots, x_{N}(s)\right)-K\left(s, u_{1}(s), u_{2}(s), \ldots, u_{N}(s)\right)\right| d s}{2}+\ldots \\
& \quad+\frac{\int_{0}^{a} B(t, s)\left|K\left(s, x_{N}(s), x_{1}(s), \ldots, x_{N-1}(s)\right)-K\left(s, u_{N}(s), u_{1}(s), \ldots, u_{N-1}(s)\right)\right| d s}{2} \\
& \quad \leq \frac{\theta\left(2\left|x_{1}(s)-u_{1}(s)\right|+\cdots+2\left|x_{N}(s)-u_{N}(s)\right|\right)}{2} \leq \frac{\theta\left(\left|g x_{1}(s)-g u_{1}(s)\right|+\cdots+\left|g x_{N}(s)-g u_{N}(s)\right|\right)}{2} \\
& \quad=\theta\left(\frac{d\left(g x_{1}, g u_{1}\right)+\cdots+d\left(g x_{N}, g u_{N}\right)}{2}\right)
\end{aligned}
$$


Take $\varphi(t)=t$ and $\psi(t)=\theta t$. We get

$$
\begin{aligned}
& \varphi\left(\frac{d\left(F\left(x_{1}, \ldots, x_{N}\right), F\left(u_{1}, \ldots, u_{N}\right)\right)+\cdots+d\left(F\left(x_{N}, \ldots, x_{N-1}\right), F\left(u_{N}, \ldots, u_{N-1}\right)\right)}{2}\right) \\
& \quad \leq \psi\left(\frac{d\left(g x_{1}, g u_{1}\right)+\cdots+d\left(g x_{N}, g u_{N}\right)}{2}\right)
\end{aligned}
$$

Thus $F$ and $g$ are $(\varphi-\psi)$-contractive. Therefore by using (C4) there exists $\left(x_{1}^{0}, x_{2}^{0}, \ldots, x_{N}^{0}\right) \in X^{N}$ such that

$$
\begin{aligned}
g x_{1}^{0} & \leq F\left(x_{1}^{0}, x_{2}^{0}, \ldots, x_{N}^{0}\right), g x_{2}^{0} \\
& \leq F\left(x_{2}^{0}, x_{3}^{0}, \ldots, x_{1}^{0}\right), \ldots, g x_{N}^{0} \\
& \leq F\left(x_{N}^{0}, x_{1}^{0}, \ldots, x_{N-1}^{0}\right) .
\end{aligned}
$$

Hence $\left(X^{N}\right)_{g}^{F} \neq \emptyset$. Hence there exists a unique N-tupled common fixed point $\left(x_{1}^{*}, x_{2}^{*}, \ldots, x_{N}^{*}\right) \in X^{N}$ of $F$ and $g$ is the unique solution of system (80).

\section{Data Availability}

No data were used to support this study.

\section{Conflicts of Interest}

The authors declare that they have no conflicts of interest.

\section{Authors' Contributions}

The authors contributed equally and significantly in writing this paper. All authors read and approved the final manuscript.

\section{Acknowledgments}

The authors extend their appreciation to the Deanship of Scientific Research at King Khalid University for funding this work through General Research Project under grant number G.R.P-53-39.

\section{References}

[1] A. H. Soliman and T. Nabil, "On the Existence of Coincidence and Common Fixed Point of Two Rational Type Contractions and an Application in Dynamical Programming," Journal of Function Spaces, vol. 2016, Article ID 3690421, 10 pages, 2016.

[2] T. NABIL and A. H. Soliman, "Weak Convergence Theorems of Explicit Iteration Process with Errors and Applications in Optimization," Journal of Analysis \& Number Theory, vol. 5, no. 1, pp. 81-89, 2017.

[3] T. Nabil and A. H. Soliman, "Weak convergence theorems of implicit iterative process with errors by metric projection methods and applications in signal analysis," Journal of Computational and Theoretical Nanoscience, vol. 14, no. 11, pp. 54915498, 2017.

[4] T. Nabil, "Coupled fixed point of firmly nonexpansive mappings by Manns iterative processes in Hilbert space," Journal of Computational Analysis and Applications, vol. 27, pp. 809-821, 2019.

[5] N. V. Luong and N. X. Thuan, "Coupled fixed point theorems for mixed monotone mappings and an application to integral equations," Computers \& Mathematics with Applications. An International Journal, vol. 62, no. 11, pp. 4238-4248, 2011.

[6] B. C. Dhage, "Hybrid fixed point theory in partially ordered normed linear spaces and applications to fractional integral equations," Differential Equations \& Applications, vol. 5, no. 2, pp. 155-184, 2013.

[7] D. Guo and V. Lakshmikantham, "Coupled fixed points of nonlinear operators with applications," Nonlinear Analysis: Theory, Methods \& Applications, vol. 11, no. 5, pp. 623-632, 1987.

[8] T. G. Bhaskar and V. Lakshmikantham, "Fixed point theorems in partially ordered metric spaces and applications," Nonlinear Analysis: Theory, Methods \& Applications, vol. 65, no. 7, pp. 13791393, 2006.

[9] J. J. Nieto and R. Rodríguez-López, "Contractive mapping theorems in partially ordered sets and applications to ordinary differential equations," Order, vol. 22, no. 3, pp. 223-239, 2005.

[10] V. Lakshmikantham and L. Ćirić, "Coupled fixed point theorems for nonlinear contractions in partially ordered metric spaces," Nonlinear Analysis. Theory, Methods \& Applications, vol. 70, no. 12, pp. 4341-4349, 2009.

[11] B. Samet and C. Vetro, "Coupled fixed point, F-invariant set and fixed point of N-order," Annals of Functional Analysis, vol. 2, pp. 46-56, 2010.

[12] M. Imded, A. H. Soliman, B. S. Choudhury, and P. Das, "On ntupled coincidence and common fixed points results in metric space," Journal of Operators, vol. 2013, Article ID 532867, 8 pages, 2013.

[13] M. Kir, E. Yolacan, and H. Kiziltunc, "Coupled fixed point theorems in complete metric spaces endowed with a directed graph and application," Open Mathematics, vol. 15, pp. 734-744, 2017.

[14] M. Abbas, H. Aydi, and E. Karapinar, "Tripled fixed points of multivalued nonlinear contraction mappings in partially ordered metric spaces," Abstract and Applied Analysis, vol. 2011, Article ID 812690, 12 pages, 2011.

[15] H. Aydi, E. Karapınar, and Z. Mustafa, "Coupled coincidence point results on generalized distance in ordered cone metric spaces," Positivity, vol. 17, no. 4, pp. 979-993, 2013.

[16] H. Aydi, E. Karapinar, and M. Postolache, "Tripled coincidence point theorems for weak $\varphi$-contractions in partially ordered metric spaces," Fixed Point Theory and Applications, vol. 2012, article 44, 2012. 
[17] H. Aydi, A. Felhi, Z. Mustafa, W. Shatanawi, and M. M. M. Jaradat, "Strong coupled fixed points for couplings via cdistances," Journal of Mathematical Analysis, vol. 9, no. 3, pp. 28-37, 2018.

[18] Z. Mustafa, H. Aydi, and E. Karapinar, "Mixed $g$-monotone property and quadruple fixed point theorems in partially ordered metric spaces," Fixed Point Theory and Applications, vol. 2012, p. 71, 2012.

[19] T. Rashid, Q. H. Khan, and H. Aydi, "On strong coupled coincidence points of g-Couplings and an application," Journal of Function Spaces, Article ID 4034535, 2018.

[20] B. Samet, E. Karapinar, H. Aydi, and V. Ć. Rajić, "Discussion on some coupled fixed point theorems," Fixed Point Theory and Applications, vol. 2013, article 50, 2013.

[21] W. Shatanawi, M. Abbas, H. Aydi, and N. Tahat, "Common coupled coincidence and coupled fixed points in G-metric spaces," Journal of Nonlinear Analysis and Application, vol. 2012, Article ID jnaa-00162, 16 pages, 2012.

[22] H. Aydi, E. Karapinar, and S. Radenovic, “Tripled coincidence fixed point results for Boyd-Wong and Matkowski type contractions," Revista de la Real Academia de Ciencias Exactas, Fisicas y Naturales. Serie A. Matematicas, vol. 107, no. 2, pp. 339-353, 2013.

[23] B. S. Choudhury and A. Kundu, "A coupled coincidence point result in partially ordered metric spaces for compatible mappings," Nonlinear Analysis. Theory, Methods \& Applications, vol. 73, no. 8, pp. 2524-2531, 2010.

[24] M. Jain, N. Gupta, and S. Kumar, "Coupled fixed point theorems for $(\phi-\psi)$-contractive mixed monotone mappings in partially ordered metric spaces and applications," Interational Journal of Analysis, vol. 2014, Article ID 586096, 9 pages, 2014.

[25] C. Chifu and G. Petrusel, "New results on coupled fixed point theory in metric spaces endowed with a directed graph," Fixed Point Theory and Applications, 2014:151, 13 pages, 2014.

[26] S. Suantai, P. Charoensawan, and T. A. Lampert, "Common coupled fixed point theorems for $\theta$ - $\psi$-contraction mappings endowed with a directed graph," Fixed Point Theory and Applications, vol. 2015, no. 1, article no. 224, pp. 1-11, 2015. 


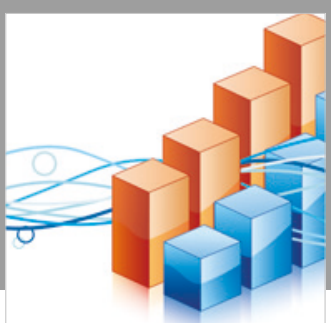

Advances in

Operations Research

\section{-n-m}
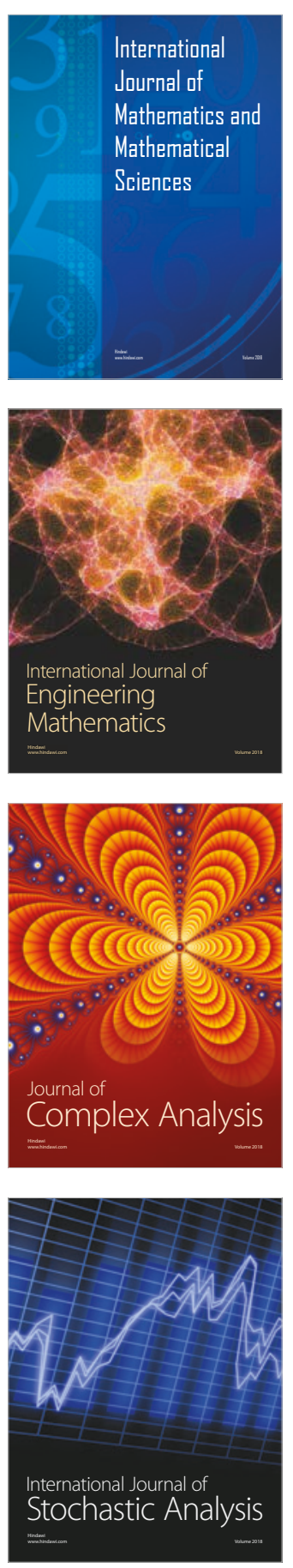
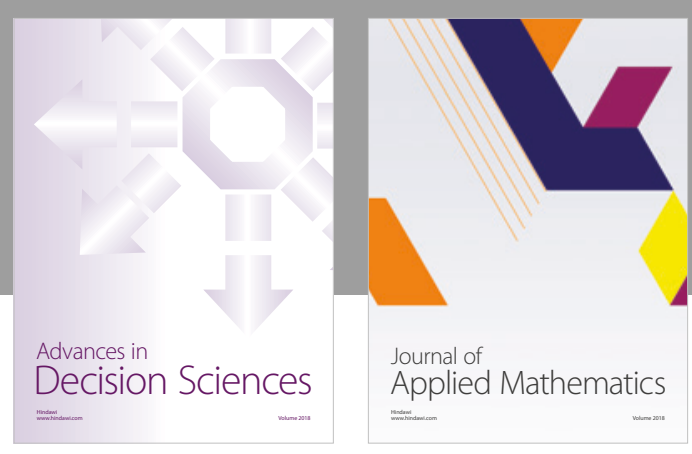

Journal of

Applied Mathematics
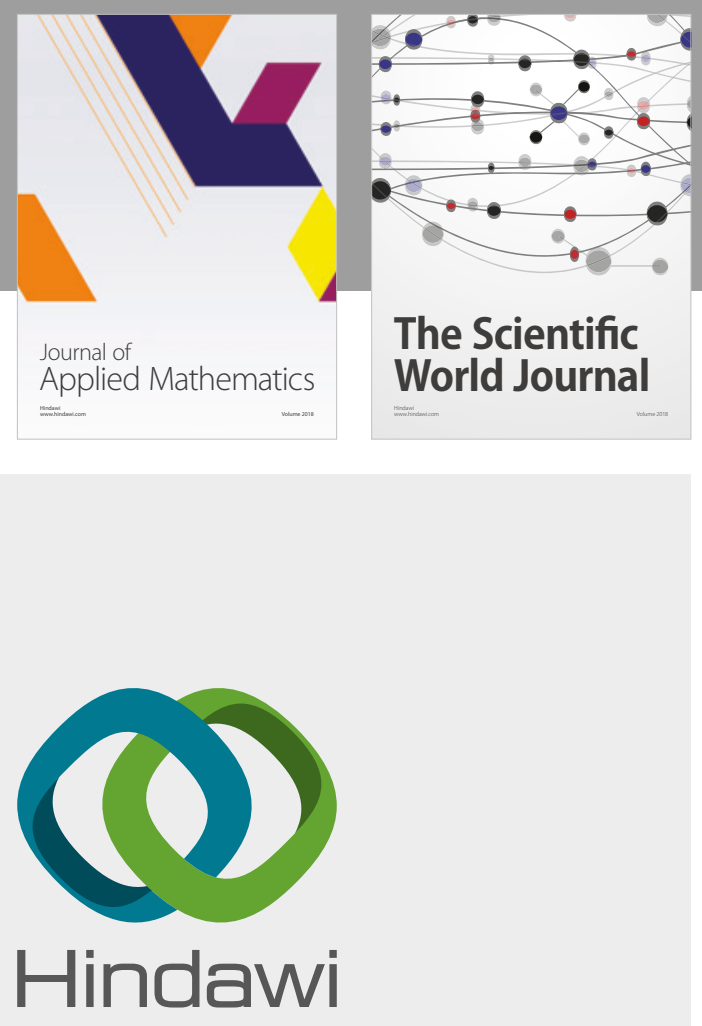

Submit your manuscripts at

www.hindawi.com

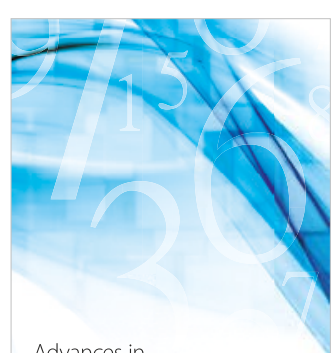

Advances in
Numerical Analysis
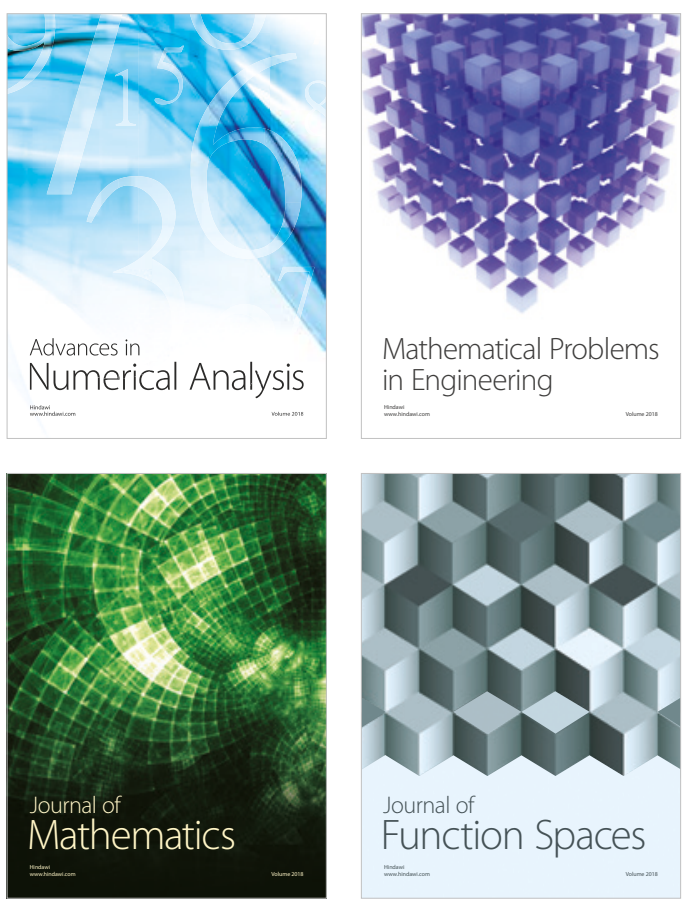

Mathematical Problems in Engineering

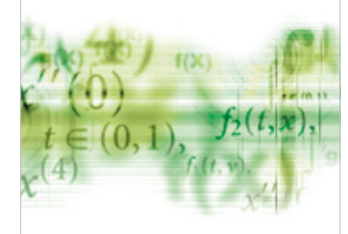

International Journal of

Differential Equations

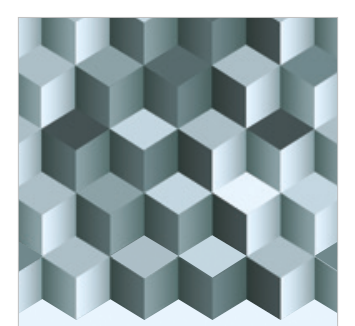

Journal of

Function Spaces

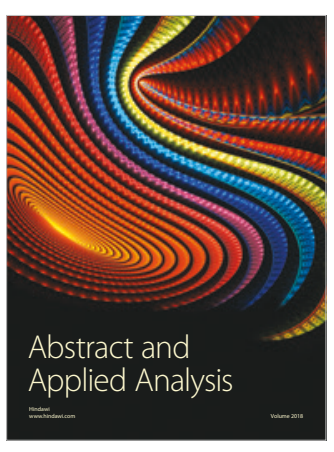

The Scientific

World Journal

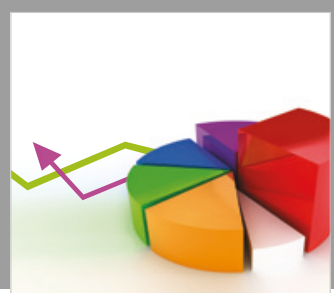

Journal of

Probability and Statistics
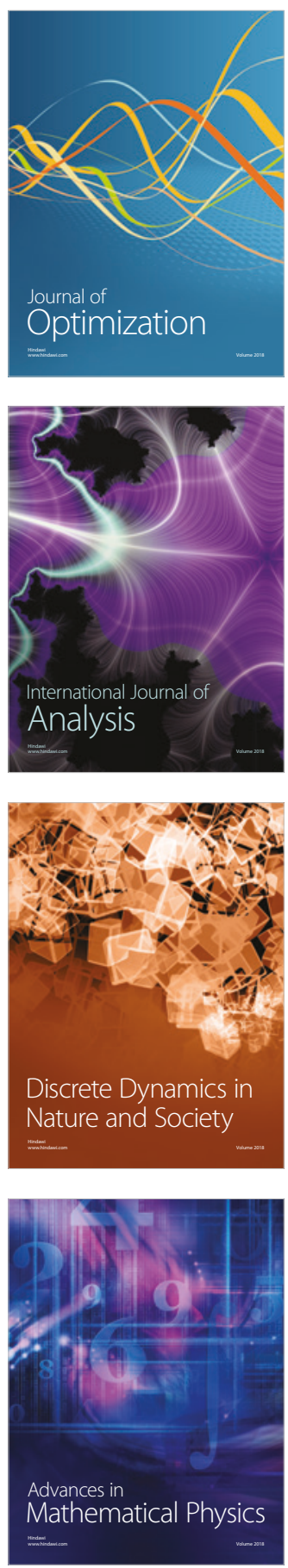